\title{
A Description Logic of Typicality for Conceptual Combination
}

\author{
Antonio Lieto* and Gian Luca Pozzato** \\ *Dipartimento di Informatica, Università di Torino and ICAR-CNR, Italy \\ **Dipartimento di Informatica, Università di Torino, Italy \\ \{antonio.lieto, gianluca.pozzato\}eunito.it
}

\begin{abstract}
We propose a nonmonotonic Description Logic of typicality able to account for the phenomenon of combining prototypical concepts, an open problem in the fields of AI and cognitive modelling. Our logic extends the logic of typicality $\mathcal{A L C}+\mathbf{T}_{\mathbf{R}}$, based on the notion of rational closure, by inclusions $p \quad:: \mathbf{T}(C) \sqsubseteq D$ ("we have probability $p$ that typical $C$ s are $D$ s"), coming from the distributed semantics of probabilistic Description Logics. Additionally, it embeds a set of cognitive heuristics for concept combination. We show that the complexity of reasoning in our logic is EXPTIME-complete as in $\mathcal{A L C}$.
\end{abstract}

\section{Introduction}

Inventing novel concepts by combining the typical knowledge of pre-existing ones is an important human creative ability. Dealing with this problem requires, from an AI perspective, the harmonization of two conflicting requirements that are hardly accommodated in symbolic systems: the need of a syntactic compositionality (typical of logical systems) and that one concerning the exhibition of typicality effects [1]. According to a well-known argument [2], in fact, prototypical concepts are not compositional. The argument runs as follows: consider a concept like pet fish. It results from the composition of the concept pet and of the concept fish. However, the prototype of pet fish cannot result from the composition of the prototypes of a pet and a fish: e.g. a typical pet is furry and warm, a typical fish is grayish, but a typical pet fish is neither furry and warm nor grayish (typically, it is red).

In this work we provide a framework able to account for this type of human-like concept combination. We propose a nonmonotonic Description Logic (from now on DL) of typicality called $\mathbf{T}^{\mathrm{CL}}$ (typical compositional logic). This logic combines two main ingredients. The first one relies on the DL of typicality $\mathcal{A L C}+\mathbf{T}_{\mathbf{R}}$ introduced in [3]. In this logic, "typical" properties can be directly specified by means of a "typicality" operator $\mathbf{T}$ enriching the underlying DL, and a TBox can contain inclusions of the form $\mathbf{T}(C) \sqsubseteq D$ to represent that "typical $C$ s are also $D s$ ". As a difference with standard DLs, in the logic $\mathcal{A L C}+\mathbf{T}_{\mathbf{R}}$ one can consistently express exceptions and reason about defeasible inheritance as well. For instance, a knowledge base can consistently express that "normally, athletes are in fit", whereas "sumo wrestlers usually are not in fit" by $\mathbf{T}$ (Athlete) $\sqsubseteq$ InFit and $\mathbf{T}$ (SumoWrestler $) \sqsubseteq \neg$ InFit, given that SumoWreslter $\sqsubseteq$ Athlete. The semantics of the $\mathbf{T}$ operator is characterized by the properties of rational logic [4], recognized as the core properties of nonmonotonic reasoning. $\mathcal{A L C}+\mathbf{T}_{\mathbf{R}}$ is characterized by a minimal model semantics corresponding to an extension to DLs of a notion of rational closure as defined in [4] for propositional logic: the idea is to adopt a preference relation among $\mathcal{A L C}+\mathbf{T}_{\mathbf{R}}$ models, where intuitively 
a model is preferred to another one if it contains less exceptional elements, as well as a notion of minimal entailment restricted to models that are minimal with respect to such preference relation. As a consequence, $\mathbf{T}$ inherits well-established properties like specificity and irrelevance: in the example, the logic $\mathcal{A L C}+\mathbf{T}_{\mathbf{R}}$ allows us to infer $\mathbf{T}($ Athlete $\sqcap$ Bald $) \sqsubseteq$ InFit (being bald is irrelevant with respect to being in fit) and, if one knows that Hiroyuki is a typical sumo wrestler, to infer that he is not in fit, giving preference to the most specific information.

As a second ingredient, we consider a distributed semantics similar to the one of probabilistic DLs known as DISPONTE [5], allowing to label axioms with degrees representing probabilities, but restricted to typicality inclusions. The basic idea is to label inclusions $\mathbf{T}(C) \sqsubseteq D$ with a real number between 0.5 and 1 , representing its probability ${ }^{1}$, assuming that each axiom is independent from each others. The resulting knowledge base defines a probability distribution over scenarios: roughly speaking, a scenario is obtained by choosing, for each typicality inclusion, whether it is considered as true or false. In a slight extension of the above example, we could have the need of representing that both the typicality inclusions about athletes and sumo wrestlers have a probability of $80 \%$, whereas we also believe that athletes are usually young with a higher probability of $95 \%$, with the following KB: (1) SumoWrestler $\sqsubseteq$ Athlete; (2) 0.8 :: $\mathbf{T}$ (Athlete) $\sqsubseteq$ InFit; (3) 0.8 :: $\mathbf{T}$ (SumoWrestler $) \sqsubseteq \neg$ InFit; (4) 0.95 :: $\mathbf{T}$ (Athlete) $\sqsubseteq$ YoungPerson. We consider eight different scenarios, representing all possible combinations of typicality inclusion: as an example, $\{((2), 1),((3), 0),((4), 1)\}$ represents the scenario in which (2) and (4) hold, whereas (3) does not. We equip each scenario with a probability depending on those of the involved inclusions, then we restrict reasoning to scenarios whose probabilities belong to a given and fixed range.

We show that the proposed logic $\mathbf{T}^{\mathrm{CL}}$ is able to tackle the problem of composing prototypical concepts. As an additional element of the proposed formalization we employ a method inspired by cognitive semantics [6] for the identification of a dominance effect between the concepts to be combined: for every combination, we distinguish a HEAD, representing the stronger element of the combination, and a MODIFIER. The basic idea is: given a KB and two concepts $C_{H}$ (HEAD) and $C_{M}$ (MODIFIER) occurring in it, we consider only some scenarios in order to define a revised knowledge base, enriched by typical properties of the combined concept $C \sqsubseteq C_{H} \sqcap C_{M}$ (the heuristics for the scenario selections are detailed in Section 2, Definition 7).

In this paper we exploit $\mathbf{T}^{\mathrm{CL}}$ by showing how it is able to capture well established examples in the literature of cognitive science concerning concept combination. On the other hand, we have also shown [7] how $\mathbf{T}^{\mathrm{CL}}$ can be also used as a tool for the generation of novel creative concepts, that could be useful in many applicative scenarios. We also show that the reasoning complexity of $\mathbf{T}^{\mathrm{CL}}$ is EXPTIME-complete, as in the standard $\mathcal{A L C}$ logic.

\section{A Logic for Concept Combination}

The nonmonotonic Description Logic $\mathbf{T}^{\mathrm{CL}}$ combines the semantics based on the rational closure of $\mathcal{A L C}+\mathbf{T}_{\mathbf{R}}$ [3] with the probabilistic DISPONTE semantics [5]. By taking

\footnotetext{
${ }^{1}$ Here, we focus on the proposal of the formalism itself, therefore the machinery for obtaining probabilities from an application domain will not be discussed.
} 
inspiration from [8], we consider two types of properties associated to a given concept: rigid and typical. Rigid properties are those defining a concept, e.g. $C \sqsubseteq D$ (all $C$ s are $D$ s). Typical properties are represented by inclusions equipped by a probability. Additionally, we employ a cognitive heuristic for the identification of a dominance effect between the concepts to be combined, distinguishing between HEAD and MODIFIER ${ }^{2}$.

The language of $\mathbf{T}^{\mathrm{CL}}$ extends the basic DL $\mathcal{A L C}$ by typicality inclusions of the form $\mathbf{T}(C) \sqsubseteq D$ equipped by a real number $p \in(0.5,1)$ representing its probability, whose meaning is that "normally, $C$ s are also $D$ with probability $p$ ".

Definition 1 (Language of $\mathbf{T}^{\mathrm{CL}}$ ). We consider an alphabet of concept names $\mathrm{C}$, of role names $\mathrm{R}$, and of individual constants $\mathrm{O}$. Given $A \in \mathrm{C}$ and $R \in \mathrm{R}$, we define:

$$
C, D:=A|\top| \perp|\neg C| C \sqcap C|C \sqcup C| \forall R . C \mid \exists R . C
$$

We define a knowledge base $\mathcal{K}=\langle\mathcal{R}, \mathcal{T}, \mathcal{A}\rangle$ where: $\bullet \mathcal{R}$ is a finite set of rigid properties of the form $C \sqsubseteq D ; \bullet \mathcal{T}$ is a finite set of typicality properties of the form $p:: \mathbf{T}(C) \sqsubseteq$ $D$, where $p \in(0.5,1) \subseteq \mathbb{R}$ is the probability of the inclusion; $\bullet \mathcal{A}$ is the ABox, i.e. $a$ finite set of formulas of the form $C(a)$ where $a \in 0$.

It is worth noticing that we avoid typicality inclusions with degree 1 . Indeed, an inclusion 1 :: $\mathbf{T}(C) \sqsubseteq D$ would mean that it is a certain property, that we represent with $C \sqsubseteq D \in \mathcal{R}$. Also, observe that we only allow typicality inclusions equipped with probabilities $p>0.5$. Indeed, the very notion of typicality derives from that one of probability distribution, in particular typical properties attributed to entities are those characterizing the majority of instances involved. Moreover, in our effort of integrating two different semantics - DISPONTE and typicality logic - the choice of having probabilities higher than 0.5 for typicality inclusions seems to be the only one compliant with both the formalisms. In fact, despite the DISPONTE semantics allows to assign also low probabilities/degrees of belief to standard inclusions, in the logic $\mathbf{T}^{\mathrm{CL}}$ it would be misleading to also allow low probabilities for typicality inclusions. Please, note that this is not a limitation of the expressivity of the logic $\mathbf{T}^{\mathrm{CL}}$ : we can in fact represent properties not holding for typical members of a category, for instance if one need to represent that typical students are not married, we can have that 0.8 :: $\mathbf{T}$ (Student) $\sqsubseteq \neg$ Married.

Following from the DISPONTE semantics, each axiom is independent from each others. This avoids the problem of dealing with probabilities of inconsistent inclusions.

A model $\mathcal{M}$ of $\mathbf{T}^{\mathrm{CL}}$ extends standard $\mathcal{A L C}$ models by a preference relation among domain elements as in the logic of typicality [3]. In this respect, $x<y$ means that $x$ is "more normal" than $y$, and that the typical members of a concept $C$ are the minimal elements of $C$ with respect to this relation. An element $x \in \Delta^{\mathcal{I}}$ is a typical instance of some concept $C$ if $x \in C^{\mathcal{I}}$ and there is no $C$-element in $\Delta^{\mathcal{I}}$ more normal than $x$.

Definition 2 (Model of $\mathbf{T}^{\mathrm{CL}}$ ). A model $\mathcal{M}$ is any structure $\left\langle\Delta^{\mathcal{I}},<,{ }^{\mathcal{I}}\right\rangle$ where: $(i) \Delta^{\mathcal{I}}$ is a non empty set of items called the domain; $(i i)<$ is an irreflexive, transitive, wellfounded and modular (for all $x, y, z$ in $\Delta^{\mathcal{I}}$, if $x<y$ then either $x<z$ or $z<y$ ) relation over $\Delta^{\mathcal{I}} ;$ (iii).$^{\mathcal{I}}$ is the extension function that maps each concept $C$ to $C^{\mathcal{I}} \subseteq \Delta^{\mathcal{I}}$, and

\footnotetext{
${ }^{2}$ Here we assume that some methods for the automatic assignment of the HEAD/MODIFER pairs are/may be available and focus on the discussion of the reasoning part.
} 
each role $R$ to $R^{\mathcal{I}} \subseteq \Delta^{\mathcal{I}} \times \Delta^{\mathcal{I}}$. For concepts of $\mathcal{A L C}, C^{\mathcal{I}}$ is defined as usual. For the $\mathbf{T}$ operator, we have $(\mathbf{T}(C))^{\mathcal{I}}=\operatorname{Min}_{<}\left(C^{\mathcal{I}}\right)$, where $\operatorname{Min}_{<}\left(C^{\mathcal{I}}\right)=\left\{x \in C^{\mathcal{I}} \mid \nexists y \in\right.$ $C^{\mathcal{I}}$ s.t. $\left.y<x\right\}$.

A model $\mathcal{M}$ can be equivalently defined by postulating the existence of a function $k_{\mathcal{M}}: \Delta^{\mathcal{I}} \longmapsto \mathbb{N}$, where $k_{\mathcal{M}}$ assigns a finite rank to each domain element [3]: the rank of $x$ is the length of the longest chain $x_{0}<\ldots<x$ from $x$ to a minimal $x_{0}$, i.e. such that there is no $x^{\prime}$ such that $x^{\prime}<x_{0}$. The rank function $k_{\mathcal{M}}$ and $<$ can be defined from each other by letting $x<y$ if and only if $k_{\mathcal{M}}(x)<k_{\mathcal{M}}(y)$.

Definition 3 (Model satisfying a KB). Let $\mathcal{K}=\langle\mathcal{R}, \mathcal{T}, \mathcal{A}\rangle$ be a KB. Given a model $\mathcal{M}=\left\langle\Delta^{\mathcal{I}},<,{ }^{\mathcal{I}}\right\rangle$, we assume that.$^{\mathcal{I}}$ is extended to assign a domain element $a^{\mathcal{I}}$ of $\Delta^{\mathcal{I}}$ to each individual constant a of 0 . We say that: (i) $\mathcal{M}$ satisfies $\mathcal{R}$ if, for all $C \sqsubseteq D \in \mathcal{R}$, we have $C^{\mathcal{I}} \subseteq D^{\mathcal{I}}$; (ii) $\mathcal{M}$ satisfies $\mathcal{T}$ if, for all $q:: \mathbf{T}(C) \sqsubseteq D \in \mathcal{T}$, we have that $\mathbf{T}(C)^{\mathcal{I}} \subseteq D^{\overline{\mathcal{I}}}$, i.e. $\operatorname{Min}_{<}\left(C^{\mathcal{I}}\right) \subseteq D^{\mathcal{I}}$; (iii) $\mathcal{M}$ satisfies $\mathcal{A}$ if, for all assertion $F \in \mathcal{A}$, if $F=\bar{C}(a)$ then $a^{\mathcal{I}} \in C^{\mathcal{I}}$, otherwise if $F=R(a, b)$ then $\left(a^{\mathcal{I}}, b^{\mathcal{I}}\right) \in R^{\mathcal{I}}$.

Even if the typicality operator $\mathbf{T}$ itself is nonmonotonic (i.e. $\mathbf{T}(C) \sqsubseteq E$ does not imply $\mathbf{T}(C \sqcap D) \sqsubseteq E)$, what is inferred from a KB can still be inferred from any KB' with $\mathrm{KB} \subseteq \mathrm{KB}$, i.e. the resulting logic is monotonic. In order to perform useful nonmonotonic inferences, in [3] the authors have strengthened the above semantics by restricting entailment to a class of minimal models. Intuitively, the idea is to restrict entailment to models that minimize the untypical instances of a concept. The resulting logic corresponds to a notion of rational closure on top of $\mathcal{A L C}+\mathbf{T}_{\mathbf{R}}$. Such a notion is a natural extension of the rational closure construction provided in [4] for the propositional logic. This nonmonotonic semantics relies on minimal rational models that minimize the rank of domain elements. Informally, given two models of $\mathrm{KB}$, one in which a given domain element $x$ has rank 2 (because for instance $z<y<x$ ), and another in which it has rank 1 (because only $y<x$ ), we prefer the latter, as in this model the element $x$ is assumed to be "more typical" than in the former. Query entailment, i.e. checking whether a formula holds in all models satisfying a $\mathrm{KB}$, is then restricted to minimal canonical models. The intuition is that a canonical model contains all the individuals that enjoy properties that are consistent with KB. This is needed when reasoning about the rank of the concepts: it is important to have them all represented. For details, see [3].

A query $F$ is minimally entailed from a $\mathrm{KB}$ if it holds in all minimal canonical models of KB. In [3] it is shown that query entailment in the nonmonotonic $\mathcal{A L C}+\mathbf{T}_{\mathbf{R}}$ is in EXPTIME.

Definition 4 (Entailment in $\mathrm{T}^{\mathrm{CL}}$ ). Let $\mathcal{K}=\langle\mathcal{R}, \mathcal{T}, \mathcal{A}\rangle$ be a $K B$ and let $F$ be either $C \sqsubseteq D\left(C\right.$ could be $\mathbf{T}\left(C^{\prime}\right)$ ) or $C(a)$ or $R(a, b)$. We say that $F$ follows from $\mathcal{K}$ if, for all minimal $\mathcal{M}$ satisfying $\mathcal{K}$, then $\mathcal{M}$ also satisfies $F$.

Let us now define the notion of scenario of the composition of concepts. Intuitively, a scenario is a knowledge base obtained by adding to all rigid properties in $\mathcal{R}$ and to all ABox facts in $\mathcal{A}$ only some typicality properties. More in detail, we define an atomic choice on each typicality inclusion, then we define a selection as a set of atomic choices in order to select which typicality inclusions have to be considered in a scenario. 
Definition 5 (Atomic choice). Given $\mathcal{K}=\langle\mathcal{R}, \mathcal{T}, \mathcal{A}\rangle$, where $\mathcal{T}=\left\{E_{1}=q_{1} \quad\right.$ : $\left.\mathbf{T}\left(C_{1}\right) \sqsubseteq D_{1}, \ldots, E_{n}=q_{n} \quad:: \mathbf{T}\left(C_{n}\right) \sqsubseteq D_{n}\right\}$ we define $\left(E_{i}, k_{i}\right)$ an atomic choice, where $k_{i} \in\{0,1\}$.

Definition 6 (Selection). Given $\mathcal{K}=\langle\mathcal{R}, \mathcal{T}, \mathcal{A}\rangle$, where $\mathcal{T}=\left\{E_{1}=q_{1} \quad:: \mathbf{T}\left(C_{1}\right) \sqsubseteq\right.$ $\left.D_{1}, \ldots, E_{n}=q_{n}:: \mathbf{T}\left(C_{n}\right) \sqsubseteq D_{n}\right\}$ and a set of atomic choices $\nu$, we say that $\nu$ is a selection if, for each $E_{i}$, one decision is taken, i.e. either $\left(E_{i}, 0\right) \in \nu$ and $\left(E_{i}\right.$, $1) \notin \nu$ or $\left(E_{i}, 1\right) \in \nu$ and $\left(E_{i}, 0\right) \notin \nu$ for $i=1,2, \ldots, n$. The probability of $\nu$ is $P(\nu)=\prod_{\left(E_{i}, 1\right) \in \nu} q_{i} \prod_{\left(E_{i}, 0\right) \in \nu}\left(1-q_{i}\right)$.

Definition 7 (Scenario). Given $\mathcal{K}=\langle\mathcal{R}, \mathcal{T}, \mathcal{A}\rangle$, where $\mathcal{T}=\left\{E_{1}=q_{1} \quad:: \mathbf{T}\left(C_{1}\right) \sqsubseteq\right.$ $\left.D_{1}, \ldots, E_{n}=q_{n} \quad:: \mathbf{T}\left(C_{n}\right) \sqsubseteq D_{n}\right\}$ and given a selection $\sigma$, we define a scenario $w_{\sigma}=\left\langle\mathcal{R},\left\{E_{i} \mid\left(E_{i}, 1\right) \in \sigma\right\}, \mathcal{A}\right\rangle$. We also define the probability of a scenario $w_{\sigma}$ as the probability of the corresponding selection, i.e. $P\left(w_{\sigma}\right)=P(\sigma)$. Last, we say that a scenario is consistent with respect to $\mathcal{K}$ when it admits a model in the logic $\mathbf{T}^{\mathcal{L}}$ satisfying $\mathcal{K}$.

We denote with $\mathcal{W}_{\mathcal{K}}$ the set of all scenarios. It follows that the probability of a scenario $P\left(w_{\sigma}\right)$ is a probability distribution over scenarios, that is to say $\sum_{w \in \mathcal{W}_{\mathcal{K}}} P(w)=1$.

Given a $\mathrm{KB} \mathcal{K}=\langle\mathcal{R}, \mathcal{T}, \mathcal{A}\rangle$ and given two concepts $C_{H}$ and $C_{M}$ occurring in $\mathcal{K}$, our logic allows defining the compound concept $C$ as the combination of the HEAD $C_{H}$ and the MODIFIER $C_{M}$, where $C \sqsubseteq C_{H} \sqcap C_{M}$ and the typical properties of the form $\mathbf{T}(C) \sqsubseteq D$ to ascribe to the concept $C$ are obtained in the set of scenarios that: i) are consistent with respect to $\mathcal{K}$; ii) are not trivial, i.e. those with the highest probability, in the sense that the scenarios considering all properties that can be consistently ascribed to $C$ are discarded; iii) are those giving preference to the typical properties of the HEAD $C_{H}$ (with respect to those of the MODIFIER $C_{M}$ ) with the highest probability.

Lastly, we define the ultimate output of our mechanism: a knowledge base in the logic $\mathbf{T}^{\mathrm{CL}}$ whose set of typicality properties is enriched by those of the compound concept $C$. Given a scenario $w$ satisfying the above properties, we define the properties of $C$ as the set of inclusions $p:: \mathbf{T}(C) \sqsubseteq D$, for all $\mathbf{T}(C) \sqsubseteq D$ that are entailed (Definition 4) from $w$ in the logic $\mathbf{T}^{\mathrm{CL}}$. The probability $p$ is such that: (i) if $D$ is a property inherited either from the HEAD or from both the HEAD and the MODIFIER, that is to say $\mathbf{T}\left(C_{H}\right) \sqsubseteq D$ is entailed from $w$, then $p$ corresponds to the probability of such inclusion in the initial knowledge base, i.e. $p: \mathbf{T}\left(C_{H}\right) \sqsubseteq D \in \mathcal{T}$; (ii) otherwise, i.e. $\mathbf{T}\left(C_{M}\right) \sqsubseteq D$ is entailed from $w$, then $p$ corresponds to the probability of such inclusion in the initial knowledge base, i.e. $p: \mathbf{T}\left(C_{M}\right) \sqsubseteq D \in \mathcal{T}$.

The knowledge base obtained as the result of combining concepts $C_{H}$ and $C_{M}$ into the compound concept $C$ is called $C$-revised knowledge base:

$$
\mathcal{K}_{C}=\langle\mathcal{R}, \mathcal{T} \cup\{p: \mathbf{T}(C) \sqsubseteq D\}, \mathcal{A}\rangle,
$$

for all $D$ such that $\mathbf{T}(C) \sqsubseteq D$ is entailed in $w$ and $p$ defined as above. Notice that, since the $C$-revised knowledge base is still in the language of the $\mathbf{T}^{\mathrm{CL}}$ logic, we can iteratively repeat the same procedure in order to combine not only atomic concepts, but also compound concepts. We leave a detailed analysis of this topic for future works. 
Let us now define the probability that a query is entailed from a $C$-revised knowledge base. We restrict our concern to ABox facts. The idea is that, given a query $A(a)$ and its probability $p$, the probability of $A(a)$ is the product of $p$ and the probability of the inclusion in the $C$-revised knowledge base which is responsible for that.

Definition 8 (Probability of query entailment). Given a knowledge base $\mathcal{K}=\langle\mathcal{R}, \mathcal{T}, \mathcal{A}\rangle$, the $C$-revised knowledge base $\mathcal{K}_{C}$, a query $A(a)$ and its probability $p \in(0,1]$, we define the probability of the entailment of $A(a)$ from $\mathcal{K}_{C}$, denoted as $\mathbb{P}(A(a), p)$ as follows:

- $\mathbb{P}(A(a), p)=0$, if $A(a)$ is not entailed from $\mathcal{K}_{C}$;

- $\mathbb{P}(A(a), p)=p \times q$, where either $q:: \mathbf{T}(C) \sqsubseteq A$ belongs to $\mathcal{K}_{C}$ or $q:: \mathbf{T}(C) \sqsubseteq D$ belongs to $\mathcal{K}_{C}$ and $D \sqsubseteq A$ is entailed from $\mathcal{R}$ in standard $\mathcal{A L C}$, otherwise.

We conclude by showing that reasoning in $\mathbf{T}^{\mathrm{C}\llcorner}$ remains in the same complexity class of standard $\mathcal{A L C}$. For the completeness, let $n$ be the size of KB, then the number of typicality inclusions is $O(n)$. It is straightforward to observe that we have an exponential number of different scenarios, for each one we need to check whether the resulting $\mathrm{KB}$ is consistent in $\mathcal{A L C}+\mathbf{T}_{\mathbf{R}}$ which is EXPTIME-complete. Hardness immediately follows form the fact that $\mathbf{T}^{\mathrm{CL}}$ extends $\mathcal{A L C}+\mathbf{T}_{\mathbf{R}}$. Reasoning in the revised knowledge base relies on reasoning in $\mathcal{A L C}+\mathbf{T}_{\mathbf{R}}$, therefore we can conclude:

Theorem 1. Reasoning in $\mathbf{T}^{c L}$ is EXPTIME-complete.

\section{Applications of the logic $\mathrm{T}^{\mathrm{CL}}$}

We propose two different types of examples adopting the logic $\mathbf{T}^{\mathrm{CL}}$ to model the phenomenon of typicality-based conceptual combination, in order to show how our logic is able to handle two paradigmatic examples of typicality-based concept composition coming from the Cognitive Science. Such cases have been historically problematic to model by adopting other kinds of logics (for example fuzzy logic, [2], [6], [9]) and have been recently considered as a testbed for computational models aiming at dealing with this type of problem [10]. The exploitation of $\mathbf{T}^{\mathrm{CL}}$ as a possible application in the field of computational creativity for the generation of new characters is proposed in [7].

\subsection{Pet Fish}

Let $\mathcal{K}=\langle\mathcal{R}, \mathcal{T}, \emptyset\rangle$ be a $\mathrm{KB}$, where $\mathcal{R}=\{$ Fish $\sqsubseteq \exists$ livesIn. Water $\}$ and $\mathcal{T}$ is:

1. 0.9 :: $\mathbf{T}($ Pet $) \sqsubseteq \exists$ livesIn. $(\neg$ Water $)$

2. $0.8:: \mathbf{T}($ Pet $) \sqsubseteq$ Affectionate

3. 0.7 :: $\mathbf{T}($ Fish $) \sqsubseteq \neg$ Affectionate

4. 0.8 :: $\mathbf{T}($ Pet $) \sqsubseteq$ Warm

5. 0.6 :: $\mathbf{T}($ Fish $) \sqsubseteq$ Greyish

6. 0.9 :: $\mathbf{T}($ Fish $) \sqsubseteq$ Scaly

7. 0.8 :: $\mathbf{T}($ Fish $) \sqsubseteq \neg$ Warm

By the properties of the typicality operator $\mathbf{T}$, we have that $(*) \quad \mathbf{T}($ Pet $\sqcap$ Fish $) \sqsubseteq$ $\exists$ livesIn.Water. Indeed, Fish $\sqsubseteq \exists$ livesIn. Water is a rigid property, which is always preferred to a typical one: in this case, additionally, the rigid property is also associated to the HEAD element fish. Therefore, this element is reinforced.

Since $|\mathcal{T}|=7$, we have $2^{7}=128$ different scenarios. We can observe that some of them are not consistent, more precisely those (i) containing the inclusion 1 by $(*)$; (ii) containing inclusions 2 and 3; (iii) containing inclusions 4 and 7 . The scenarios with the 
highest probabilities (up to 17\%) are both trivial and inconsistent: indeed, probabilities $p_{i}$ equipping typicality inclusions are such that $p_{i}>0.5$, therefore the higher is the number of inclusions belonging to a scenario the higher is the associated probability. Since typicality inclusions introduce properties that are pairwise inconsistent, it follows that such scenarios must be discarded.

Consistent scenarios with the highest probabilities (from $0.14 \%$ to $0.21 \%$ ) contain 2 and do not contain 3, namely they privilege the MODIFIER with respect to the corresponding negation in the HEAD, obtaining that being affectionate is a typical property of a pet fish. These scenarios are consistent, but they are trivial, since they include either all the properties associated to the MODIFIER or those with the highest probability. In both cases, in these scenarios we pay the price of discarding some properties of the HEAD. For the same reason we discard the scenarios with probabilities $0.12 \%$ containing 4 and not 7 . The remaining scenarios privilege the properties associated to the HEAD, namely they contain 3 and do not contain 2 and 7 and not 4 . Again, these scenarios range from the most trivial to the most surprising ones. It turns out that the scenarios able to account for the phenomenon of conceptual composition are not those with the highest probability, but those belonging to the probability range immediately lower. In the example, most trivial scenarios where we have that typical pet fishes are greyish (probability $0.12 \%$ ). We have two remaining scenarios whose probability is immediately lower $(0.08 \%)$ : in both, we have that a typical pet fish is scaly and not affectionate, whereas they differ about being warm or not. The scenario giving preference to the inclusions associated to the HEAD, i.e. containing 7 and not 4 , is such that a typical pet fish is not warm, and it is preferred to the one including 4 and not 7 . The not trivial scenario defining prototypical properties of a pet fish comes from the selection $\sigma=\{(1,0),(2,0),(3,1),(4,0),(5,0),(6,1),(7,1)\}$, and contains 3,6 , and 7 :

$$
\begin{aligned}
& 0.8:: \mathbf{T}(\text { Pet } \sqcap \text { Fish }) \sqsubseteq \neg \text { Affectionate } \\
& 0.6:: \mathbf{T}(\text { Pet } \sqcap \text { Fish }) \sqsubseteq \text { Scaly } \\
& 0.8:: \mathbf{T}(\text { Pet } \sqcap \text { Fish }) \sqsubseteq \neg \text { Warm }
\end{aligned}
$$

Notice that in $\mathbf{T}^{\mathrm{C}}$, adding a new inclusion $\mathbf{T}($ Pet $\sqcap$ Fish $) \sqsubseteq$ Red, would not be problematic (i.e. our logic tackles the phenomenon of prototypical attributes emergence [6]).

\subsection{Linda the feminist bank teller}

We exploit the logic $\mathbf{T}^{\mathrm{CL}}$ in order to tackle the so called conjunction fallacy problem [11]: Linda is 31 years old, single, outspoken, and bright. She majored in philosophy and was concerned with issues of discrimination and social justice, and also participated in anti-nuclear demonstrations. When asked to rank the probability of the statements 1) "Linda is a bank teller" and 2) "Linda is a bank teller and is active in the feminist movement", the majority of people rank 2) as more probable than 1), violating the classic probability rules. In $\mathrm{T}^{\mathrm{CL}}$, let $\mathcal{K}=\langle\mathcal{R}, \mathcal{T}, \mathcal{A}\rangle$ be a $\mathrm{KB}$, where $\mathcal{A}=\emptyset, \mathcal{T}$ is:

$0.8:: \mathbf{T}$ (Feminist) $\sqsubseteq$ Bright

0.9 :: $\mathbf{T}$ (Feminist $) \sqsubseteq$ OutSpoken

0.8 :: $\mathbf{T}$ (Feminist $) \sqsubseteq \exists$.fightsFor SocialJustice

0.9 :: $\mathbf{T}($ Feminist $) \sqsubseteq$ Environmentalist 
0.6 :: $\mathbf{T}($ BankTeller $) \sqsubseteq \neg \exists$.fightsFor SocialJustice

0.8 :: $\mathbf{T}($ BankTeller $) \sqsubseteq$ Calm

and $\mathcal{R}$ is as follows: $\{$ BankTeller $\sqsubseteq \exists i s E m p l o y e d . B a n k$, Feminist $\sqsubseteq \exists$ believesIn.Feminism, Feminist $\sqsubseteq$ Female, Environmentalist $\sqsubseteq \exists$ isAgainst.NuclearEnergyDevelopment $\}$. Let us consider the compound concept Feminist $\sqcap$ BankTeller. It can be obtained in two different ways, namely by choosing Feminist as the HEAD and BankTeller as the MODIFIER, or vice versa. In $\mathbf{T}^{\mathrm{L}}$, the compound concept inherits all the rigid properties, that is to say Feminist $\sqcap$ BankTeller is included in $\exists$ isEmployed.Bank, in $\exists$ believesIn.Feminism and in Female. Concerning the typical properties, two of them are in contrast, namely typical feminists fight for social justice, whereas typical bank tellers do not. All the scenarios including both $\mathbf{T}$ (Feminist $) \sqsubseteq \exists$ fightsFor.SocialJustice and $\mathbf{T}($ BankTeller $) \sqsubseteq \neg \exists$ fightsFor.SocialJustice are then inconsistent. Concerning the remaining ones, if we consider Feminist as the HEAD, then scenarios including $\mathbf{T}($ BankTeller $) \sqsubseteq \neg \exists$ fightsFor.SocialJustice are discarded, in favor of scenarios including $\mathbf{T}$ (Feminist) $\sqsubseteq \exists$ fightsFor.SocialJustice. The most obvious scenario, with the highest probability, corresponds to the one including all the typicality inclusions related to the HEAD. In the logic $\mathbf{T}^{\mathrm{CL}}$ we discard it and we focus on the remaining ones. Among them, one of the scenarios having the highest probability is the one not including $\mathbf{T}$ (Feminist) $\sqsubseteq$ Bright. This scenario defines the following Feminist $\sqcap$ BankTeller-revised knowledge base:

0.9 :: $\mathbf{T}$ (Feminist $\sqcap$ BankTeller $) \sqsubseteq$ OutSpoken

0.8 :: $\mathbf{T}$ (Feminist $\sqcap$ BankTeller $) \sqsubseteq \exists$ fightsFor.SocialJustice

0.9 :: $\mathbf{T}($ Feminist $\sqcap$ BankTeller $) \sqsubseteq$ Environmentalist

0.8 :: $\mathbf{T}$ (Feminist $\sqcap$ BankTeller $) \sqsubseteq$ Calm

Let us now consider the case of the instance Linda, described as: Young Woman (linda), $\exists$ graduatedIn.Philosophy(linda), Outspoken(linda), Bright(linda), Single(linda), $\exists$ fightsFor.SocialJustice (linda), $\exists$ isAgainst.NuclearEnergyDevelopment(linda). In our logic, solving the conjunction fallacy problem means that we have to find the most appropriate category for Linda. In our case the choice is between BankTeller and Feminist $\sqcap$ BankTeller. We can assume that, in absence of any other information, the described properties that are explicitly assigned to the instance Linda can be set to a default probability value of 0.6 . Let us first consider the Feminist $\sqcap$ BankTellerrevised knowledge base, with an $\mathrm{ABox}$ asserting that Linda is a bank teller, i.e. $\mathcal{A}_{1}=$ $\{$ BankTeller $($ linda $)\}$, and let us consider each property of the instance Linda and the associated probability of entailment. None of such properties are entailed by the Feminist $\sqcap$ BankTeller-revised knowledge base with $\mathcal{A}_{1}$, therefore, for each property of the form $D($ linda $)$ we have $\mathbb{P}(D($ linda $), 0.6)=0$ (by Definition 8$)$. On the other hand, let us consider an ABox asserting that Linda is a feminist bank teller, i.e. $\mathcal{A}_{2}=\{($ Feminist $\sqcap$ BankTeller $)($ linda $)\}$. We have: (i) YoungWoman (linda) is not entailed from the Feminist $\square$ BankTeller-revised knowledge base, therefore $\mathbb{P}($ YoungWoman $($ linda $), 0.6)=0$; the same for $\exists$ graduatedIn.Philosophy (linda $)$ and Single(linda); (ii) Outspoken (linda) is entailed from the Feminist $\sqcap$ BankTellerrevised KB with $\mathcal{A}_{2}$, then, by Definition 8, we have $\mathbb{P}($ Outspoken (linda), 0.6$)=0.6 \times$ $0.9=0.54$, where 0.9 is the probability of $\mathbf{T}($ Feminist $\sqcap$ BankTeller $) \sqsubseteq$ OutSpoken in the Feminist $\sqcap$ BankTeller-revised KB; (iii) the same for $\exists$ fightsFor.SocialJustice 
(linda) entailed by using $\mathcal{A}_{2}$ : in this case, we have $\mathbb{P}(\exists$ fightsFor.SocialJustice (linda), $0.6)=0.6 \times 0.8=0.48$; (iv) $\exists$ isAgainst.NuclearEnergyDevelopment (linda) is entailed by using $\mathcal{A}_{2}$. Observe that Environmentalist $\sqsubseteq$ NuclearEnergyDevelopment follows from $\mathcal{R}$ in standard $\mathcal{A L C}$, then $\mathbb{P}(\exists$ isAgainst.NuclearEnergyDevelopment $($ linda $), 0.6)=0.6 \times 0.9=0.54$ by Definition 8 , where 0.9 is the probability of $\mathbf{T}$ (Feminist $\sqcap$ BankTeller $) \sqsubseteq$ Environmentalist in the Feminist $\sqcap$ BankTellerrevised KB. Computing the sum of the probabilities of the queries of all facts about Linda, we obtain $0.54+0.48+0.54=1.56$, to witness that the choice of $\mathcal{A}_{2}$ is more appropriate w.r.t. the choice of $\mathcal{A}_{1}$ where the sum is 0 . This means that, in our logic, the human choice of classifying Linda as a feminist bank teller sounds perfectly plausible and has to be preferred to the alternative one of classifying her as a bank teller.

\section{Related and Future Works}

Lewis and Lawry [10] present a detailed analysis of the limits of the AI approaches (i.e. set-theory, fuzzy logics, vector-space models and quantum probability) proposed to model the phenomenon of prototypical concept composition. In addition, they show how hierarchical conceptual spaces allow to model this phenomenon. While we agree with the authors about the comments moved to the described approaches, we showed that our logic can equally model, in a cognitively compliant-way, the composition of prototypes by using a computationally effective nonmonotonic DL formalism. The logic $\mathbf{T}^{\mathrm{CL}}$ also extends the works of $[12,13]$ in that it provides a set of mechanisms allowing to block inheritance of prototypical properties in concept combination.

Other attempts similar to the one proposed here, and employing the $\mathcal{E L}^{++}$[14], concerns the conceptual blending: a task where the obtained concept is entirely novel and has no strong association with the two base concepts (while, in concept combination, the compound concept is always a subset of the base concepts, for the differences see [15]). A technical difference of our work w.r.t [14] is in that what the authors call prototypes are expressed in a monotonic DL, which does not allow reasoning about typicality.

More recently, a different approach is proposed in [16], where the authors see the problem of concept blending as a nonmonotonic search problem and proposed to use Answer Set Programming (ASP) to deal with this search problem in a nonmonotonic way. Also in this case the authors propose a framework for the conceptual blending task and not for the concept combination.

As future research we aim at extending our approach to more expressive DLs. Starting from the work of [17], applying the logic with the typicality operator and the rational closure to $\mathcal{S H I} \mathcal{Q}$, we intend to study whether and how $\mathbf{T}^{\mathrm{CL}}$ could provide an alternative solution to the problem of the "all or nothing" behavior of rational closure w.r.t. property inheritance. We also aim at implementing efficient reasoners for our logic, relying on the prover RAT-OWL [18] which allows to reason in the underlying $\mathcal{A L C}+\mathbf{T}_{\mathbf{R}}$.

Acknowledgements. This work is partially supported by the project "ExceptionOWL", Università di Torino and Compagnia di San Paolo, call 2014 "Excellent (young) PI". 


\section{References}

1. Frixione, M., Lieto, A.: Representing concepts in formal ontologies: Compositionality vs. typicality effects. Logic and Logical Philosophy 21(4) (2012) 391-414

2. Osherson, D.N., Smith, E.E.: On the adequacy of prototype theory as a theory of concepts. Cognition 9(1) (1981) 35-58

3. Giordano, L., Gliozzi, V., Olivetti, N., Pozzato, G.L.: Semantic characterization of Rational Closure: from Propositional Logic to Description Logics. Artificial Intelligence 226 (2015) $1-33$

4. Lehmann, D., Magidor, M.: What does a conditional knowledge base entail? Artificial Intelligence 55(1) (1992) 1-60

5. Riguzzi, F., Bellodi, E., Lamma, E., Zese, R.: Reasoning with probabilistic ontologies. In Yang, Q., Wooldridge, M., eds.: Proceedings of the Twenty-Fourth International Joint Conference on Artificial Intelligence, IJCAI 2015, Buenos Aires, Argentina, July 25-31, 2015, AAAI Press (2015) 4310-4316

6. Hampton, J.A.: Inheritance of attributes in natural concept conjunctions. Memory \& Cognition 15(1) (1987) 55-71

7. Lieto, A., Pozzato, G.L.: Creative concept generation by combining description logic of typicality, probabilities and cognitive heuristics. In: Proceedings of the 17th International Conference of the Italian Association for Artificial Intelligence AI*IA 2018. (2018)

8. Lieto, A., Minieri, A., Piana, A., Radicioni, D.P.: A knowledge-based system for prototypical reasoning. Connection Science (27) (2015) 137-152

9. Hampton, J.A.: Conceptual combinations and fuzzy logic. Concepts and fuzzy logic 209 (2011)

10. Lewis, M., Lawry, J.: Hierarchical conceptual spaces for concept combination. Artificial Intelligence 237 (2016) 204-227

11. Tversky, A., Kahneman, D.: Extensional versus intuitive reasoning: The conjunction fallacy in probability judgment. Psychological review 90(4) (1983) 293

12. Pozzato, G.L.: Reasoning in description logics with typicalities and probabilities of exceptions. In Antonucci, A., Cholvy, L., Papini, O., eds.: Symbolic and Quantitative Approaches to Reasoning with Uncertainty - 14th European Conference, ECSQARU 2017, Lugano, Switzerland, July 10-14, 2017, Proceedings. Volume 10369 of Lecture Notes in Computer Science., Springer (2017) 409-420

13. Pozzato, G.L.: Reasoning about plausible scenarios in description logics of typicality. Intelligenza Artificiale 11(1) (2017) 25-45

14. Confalonieri, R., Schorlemmer, M., Kutz, O., Peñaloza, R., Plaza, E., Eppe, M.: Conceptual blending in EL++. In Lenzerini, M., Peñaloza, R., eds.: Proceedings of the 29th International Workshop on Description Logics, Cape Town, South Africa, April 22-25, 2016. Volume 1577 of CEUR Workshop Proceedings., CEUR-WS.org (2016)

15. Nagai, Y., Taura, T.: Formal description of concept-synthesizing process for creative design. Design computing and cognition06 (2006) 443-460

16. Eppe, M., Maclean, E., Confalonieri, R., Kutz, O., Schorlemmer, M., Plaza, E., Kühnberger, K.U.: A computational framework for conceptual blending. Artificial Intelligence 256 (2018) $105-129$

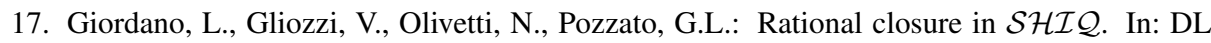
2014, 27th International Workshop on Description Logics. Volume 1193 of CEUR Workshop Proceedings., CEUR-WS.org (2014) 543-555

18. Giordano, L., Gliozzi, V., Pozzato, G.L., Renzulli, R.: An efficient reasoner for description logics of typicality and rational closure. In Artale, A., Glimm, B., Kontchakov, R., eds.: Proceedings of the 30th International Workshop on Description Logics, Montpellier, France, July 18-21, 2017. Volume 1879 of CEUR Workshop Proceedings., CEUR-WS.org (2017) 